\title{
Uniqueness and stability of solutions without the boundary condition
}

\section{Huashui Zhan ${ }^{1 *}$}

\section{"Correspondence:}

huashuizhan@163.com

'School of Applied Mathematics,

Xiamen University of Technology,

Xiamen, P.R. China

\begin{abstract}
A parabolic equation related to the $p$-Laplacian is considered. If the equation is degenerate on the boundary, then demonstrating the regularity on the boundary is difficult, the trace on the boundary cannot be defined, in general. The existence and uniqueness of weak solutions are researched. Based on uniqueness, the stability of solutions can be proved without any boundary condition.
\end{abstract}

MSC: 35K65; 35K55; 35R35

Keywords: $p$-Laplacian; Existence; Uniqueness; Stability

\section{Introduction and main results}

Consider a parabolic equation related to the $p$-Laplacian

$$
u_{t}=\operatorname{div}\left(a(u, x, t)|\nabla u|^{p-2} \nabla u\right)+\sum_{i=1}^{N} \frac{\partial b_{i}(u)}{\partial x_{i}}, \quad(x, t) \in Q_{T}=\Omega \times(0, T),
$$

with the initial value

$$
u(x, 0)=u_{0}(x), \quad x \in \Omega,
$$

where $\Omega$ is a bounded domain in $\mathbb{R}^{N}$ with appropriately smooth boundary, $p>1, u_{0}(x)$ is a $C_{0}^{1}(\Omega)$ function, $a(u, x, t) \geq 0$. If $a(u, x, t)=1$, Eq. (1.1) is the evolutionary $p$-Laplacian equation with a convective term

$$
u_{t}=\operatorname{div}\left(|\nabla u|^{p-2} \nabla u\right)+\sum_{i=1}^{N} \frac{\partial b_{i}(u)}{\partial x_{i}}, \quad(x, t) \in Q_{T}
$$

and the usual boundary condition

$$
u(x, t)=0, \quad(x, t) \in \partial \Omega \times(0, T),
$$

can be imposed. The initial-boundary boundary value problem of Eq. (1.3) has been studied in many monographs or textbooks, one can refer to [1-3] and the references therein.

(c) The Author(s) 2019. This article is distributed under the terms of the Creative Commons Attribution 4.0 International License (http://creativecommons.org/licenses/by/4.0/), which permits unrestricted use, distribution, and reproduction in any medium, provided you give appropriate credit to the original author(s) and the source, provide a link to the Creative Commons license, and indicate if changes were made. 
Benedikt et al. $[4,5]$ had studied the equation

$$
u_{t}=\operatorname{div}\left(|\nabla u|^{p-2} \nabla u\right)+q(x)\left|u^{\alpha-1}\right| u, \quad(x, t) \in Q_{T},
$$

with $0<\alpha<1$, and such that there exists an $x_{0} \in \Omega$ satisfying $q\left(x_{0}\right)>0$. They showed that the uniqueness of a solution does not hold. Meanwhile, the author of [6] had studied the equation

$$
u_{t}=\operatorname{div}\left(d^{\alpha}|\nabla u|^{p-2} \nabla u\right)+f(u, x, t), \quad(x, t) \in Q_{T},
$$

with $\alpha>0$, and has shown that the stability of solutions can be proved without any boundary condition, where $d=d(x)=\operatorname{dist}(x, \partial \Omega)$ is the distance function from the boundary and $f(s, x, t)$ is a Lipschitz function. Certainly, $|u|^{\alpha-1} u$ is not a Lipschitz function with respect to $u$, the result of [6] is compatible with those of $[4,5]$. But then, the result of [6] shows that the degeneracy of the coefficient $d^{\alpha}$ can eliminate the action from the source term $f(u, x, t)$. Moreover, we have shown that a weak solution to the equation

$$
u_{t}=\operatorname{div}\left(d^{\alpha}|\nabla u|^{p-2} \nabla u\right)+\sum_{i=1}^{N} \frac{\partial b_{i}(u)}{\partial x_{i}}, \quad(x, t) \in Q_{T},
$$

is unique independent of the boundary value condition [7], and the stability of the weak solutions can be proved in some cases [8].

For a degenerate parabolic equation, the phenomenon that the solution is free from the limitation of the boundary condition has been studied for a long time, one can refer to [915]. Roughly speaking, instead of the whole boundary condition (1.4), we may conjecture that only a partial boundary condition

$$
u(x, t)=0, \quad(x, t) \in \Sigma_{1} \times(0, T)
$$

should be imposed, where $\Sigma_{1}$ is a relatively open subset of $\partial \Omega$. In this paper, we will show that a weak solution to Eq. (1.1) is unique independent of the boundary value condition. In other words, the degeneracy of the diffusion $a(\cdot, x, t)$ on the boundary can take place regardless of the boundary value condition.

To simplify exposition, in what follows, we assume that

$$
a(u, x, t)=\rho(x)|u|^{r},
$$

where $r>0$ is a constant, $\rho(x)$ is a $C^{1}(\bar{\Omega})$ nonnegative function and

$$
\left.\rho(x)\right|_{x \in \partial \Omega}=0,\left.\quad \rho(x)\right|_{x \in \Omega}>0 .
$$

Let

$$
u=|v|^{\beta-1} v, \quad \beta=\frac{p-1}{r+p-1}, \quad \delta=\beta^{p-1} .
$$


Then Eq. (1.1) becomes

$$
\left(|v|^{\beta-1} v\right)_{t}=\delta \operatorname{div}\left(\rho(x)|\nabla v|^{p-2} \nabla v\right)+\sum_{i=1}^{N} \frac{\partial a_{i}(v)}{\partial x_{i}},
$$

where

$$
a_{i}(v)=b_{i}\left(|v|^{\beta-1} v\right) .
$$

The initial value matching up to Eq. (1.7) is

$$
|v|^{\beta-1} v(x, 0)=u_{0}(x), \quad x \in \Omega .
$$

Definition 1.1 Function $v(x, t)$ is said to be a weak solution of Eq. (1.7) with the initial value (1.8), if $v$ satisfies

$$
v \in L_{\mathrm{loc}}^{\infty}\left(0, T ; W^{1, p}\right), \quad v \in W_{\mathrm{loc}}^{1,2}\left((0, T), L^{2}(\Omega)\right), \quad \rho|\nabla v|^{p} \in L^{1}\left(Q_{T}\right),
$$

and, for any function $\phi(x, t) \in C_{0}^{1}\left(Q_{T}\right)$, there holds

$$
\begin{aligned}
& \iint_{Q_{T}}\left(-|v|^{\beta-1} v \phi_{t}\right) d x d t+\delta \iint_{Q_{T}} \rho(x)|\nabla v|^{p-2} \nabla v \cdot \nabla \phi d x d t \\
& \quad+\sum_{i=1}^{N} \iint_{Q_{T}} a_{i}(v) \phi_{x_{i}} d x d t=0 .
\end{aligned}
$$

If $v$ is a weak solution of Eq. (1.7) with the initial value (1.8), then we say that $u=|v|^{\beta-1} v$ is a weak solution of Eq. (1.1) with the initial value (1.2).

We will give a basic result of the existence of a weak solution.

Theorem 1.2 If $p \geq 2, u_{0}(x) \geq 0,\left.\rho(x)\right|_{x \in \partial \Omega}=0$ and $\int_{\Omega} \rho(x)^{-\frac{2}{p-2}} d x<\infty$, for any given $i \in$ $\{1,2, \ldots, N\}, a_{i}(s)$ is a $C^{1}$ function and there exist constants $\alpha$ and $c$ such that

$$
\left|a_{i}(s)\right| \leq c|s|^{1+\alpha}, \quad\left|a_{i}^{\prime}(s)\right| \leq c|s|^{\alpha},
$$

then there is a nonnegative weak solution of Eq. (1.7) with the initial value (1.8) in the sense of Definition 1.1.

This theorem may not be optimal, the conditions $p \geq 2, \int_{\Omega} \rho(x)^{-\frac{2}{p-2}} d x<\infty$ and (1.11) may all be weakened. However, the main aim of this paper is to probe the uniqueness and stability of weak solutions, the main results of our paper are the following theorems.

Theorem 1.3 Let $p>1, a_{i}(s)$ be a Lipschitz function, and let $\rho(x)$ satisfy (1.6). Then a weak solution of Eq. (1.7) with the initial value (1.8) is unique. 
Theorem 1.4 Let $u(x, t)$ and $v(x, t)$ be two weak solutions of Eq. (1.7) with different initial values $u_{0}(x)$ and $v_{0}(x)$, respectively. If $p \geq 2$,

$$
\int_{\Omega} \rho(x)^{-\frac{1}{p-1}} d x \leq c
$$

and $a_{i}(s)$ is a Lipschitz function, then

$$
\begin{aligned}
& \left.\int_{\Omega}|| u\right|^{\beta-1} u(x, t)-|v|^{\beta-1} v(x, t) \mid d x \\
& \quad \leq\left.\int_{\Omega}|| u_{0}\right|^{\beta-1} u_{0}(x)-\left|v_{0}\right|^{\beta-1} v_{0}(x) \mid d x, \quad \forall t \in[0, T) .
\end{aligned}
$$

It is well-known that the usual evolutionary $p$-Laplacian equation needs to be subjected to the whole boundary condition (1.4) [2, 3]. Clearly, condition $\left.a(u, x, t)\right|_{x \in \partial \Omega}=0$ excludes the usual evolutionary $p$-Laplacian equation, while condition (1.12) excludes the conservation law equation. The uniqueness of solutions for a conservation law equation only holds in the sense of the entropy solution [2]. The equations considered in [6-8, 16-19], as well as Eq. (1.1), have apparently different characteristics from both the usual evolutionary $p$-Laplacian equation and the conservation term. Roughly speaking, in the interior of $\Omega$, Eq. (1.1) has the characteristic of the usual evolutionary $p$-Laplacian equation, while on the boundary $\partial \Omega$, Eq. (1.1) has the characteristic of the conservation law equation. Comparing with our previous works $[7,17-19]$ and $[6,8]$, the main difficulty comes from the nonlinearity of the diffusion coefficient $a(u, x, t)$. Moreover, unlike our previous works, the stability of the weak solutions is based on the uniqueness of the weak solution.

Theorem 1.3 shows that the uniqueness of the weak solution holds independently of the boundary value condition. Once we have the uniqueness of the weak solution, Theorem 1.4 shows that the stability of the weak solutions is also true without the boundary value condition. Accordingly, Theorems 1.3 and 1.4 show that not only the degeneracy of the coefficient $a(u, x, t)$ can eliminate the action from the source term $f(u, x, t)$ [6], but it may also eliminate the action of the convection term $\sum_{i=1}^{N} \frac{\partial a_{i}(v)}{\partial x_{i}}$.

\section{Existence of a solution}

Consider an approximate problem of Eq. (1.7), namely

$$
\left(|v|^{\beta-1} v\right)_{t}=\delta \operatorname{div}\left((\rho(x)+\varepsilon)|\nabla v|^{p-2} \nabla v\right)+\sum_{i=1}^{N} \frac{\partial a_{i}(v)}{\partial x_{i}},
$$

with the initial boundary value conditions

$$
\begin{aligned}
& |v|^{\beta-1} v(x, 0)=u_{0}(x), \quad x \in \Omega, \\
& v(x, t)=0, \quad(x, t) \in \partial \Omega \times(0, T) .
\end{aligned}
$$

Definition 2.1 A function $v(x, t)$ is said to be a weak solution of problem (2.1)-(2.3), if $v$ satisfies

$$
v \in L_{\mathrm{loc}}^{\infty}\left(0, T ; W_{0}^{1, p}(\Omega)\right), \quad v \in W_{\mathrm{loc}}^{1,2}\left((0, T), L^{2}(\Omega)\right),
$$


and for any $\phi(x) \in C_{0}^{1}\left(Q_{T}\right)$, there holds

$$
\begin{aligned}
& -\iint_{Q_{T}}|v|^{\beta-1} v \phi_{t} d x d t+\delta \iint_{Q_{T}}(\rho(x)+\varepsilon)|\nabla v|^{p-2} \nabla v \cdot \nabla \phi d x d t \\
& \quad+\iint_{Q_{T}} a_{i}(v) \phi_{x_{i}} d x d t=0 .
\end{aligned}
$$

For any $k>0$, we define $\varphi_{k}^{+}(s)=\beta s^{\beta-1}$ when $s \geq k^{-1}, \varphi_{k}^{+}(s)=\beta\left(a_{k} s^{2}+b_{k} s\right)$ when $0 \leq s<$ $k^{-1}$, where

$$
a_{k}=k^{2-\beta}, \quad b_{k}=k^{1-\beta} \frac{3-\beta}{2}, \quad k=1,2, \ldots
$$

Extending $\varphi^{+}(s)$ to be an even function on the whole $\mathbb{R}^{1}$, and denoting it as $\varphi_{k}(s)$, we have $\varphi_{k}(s) \in C^{1}, \varphi_{k}(s) \rightarrow \beta s^{\beta-1}, s \neq 0$ as $k \rightarrow \infty$. By considering the following approximate problem:

$$
\begin{aligned}
& \varphi_{k}(v) v_{t}=\delta \operatorname{div}\left((\rho(x)+\varepsilon)\left(|\nabla v|^{2}+\frac{1}{k}\right)^{\frac{p-2}{2}} \nabla v\right)+\sum_{i=1}^{N} \frac{\partial a_{i}(v)}{\partial x_{i}}, \\
& v_{k}(x, t)=0, \quad(x, t) \in \partial \Omega \times(0, T) \\
& v_{k}(x, 0)=v_{0 k}(x), \quad x \in \Omega
\end{aligned}
$$

where $\left\|v_{0 k}(x)-v_{0}(x)\right\|_{p} \rightarrow 0$ as $k \rightarrow 0$ and $|v|^{\beta-1} v_{0}(x)=u_{0}(x)$, we obtain that there is a unique classical solution $v_{k \varepsilon}$ of problem (2.6)-(2.8). Let $k \rightarrow \infty$. Similarly as in [20], we can prove that

$$
v_{k \varepsilon} \rightarrow v_{\varepsilon}, \quad \text { a.e. in } Q_{T},
$$

and $v_{\varepsilon}$ is a solution of problem (2.1)-(2.3) in the sense of Definition 2.1; we omit the details here. In particular, if $u_{0}(x) \in L^{\infty}(\Omega)$, then we have

$$
\left\|v_{k \varepsilon}\right\|_{L^{\infty}\left(Q_{T}\right)} \leq c, \quad\left\|v_{\varepsilon}\right\|_{L^{\infty}\left(Q_{T}\right)} \leq c
$$

where $c$ is a constant independent of $k$ and $\varepsilon$, but depending on $\left\|u_{0}\right\|_{L^{\infty}(\Omega)}$. In what follows, we call $v_{\varepsilon}$ an asymptotic solution.

Proof of Theorem 1.2 Multiplying (2.1) by $v_{\varepsilon}$ and integrating over $Q_{T}$, we have

$$
\begin{aligned}
& \frac{\beta}{\beta+1} \int_{\Omega} v_{\varepsilon}^{\beta+1} d x+\delta \iint_{Q_{T}} \rho_{\varepsilon}\left|\nabla v_{\varepsilon}\right|^{p} d x d t+\sum_{i=1}^{N} \iint_{Q_{T}} v_{\varepsilon} \frac{\partial a_{i}\left(v_{\varepsilon}\right)}{\partial x_{i}} d x d t \\
& =\frac{\beta}{\beta+1} \int_{\Omega} v_{0}^{\beta+1} d x
\end{aligned}
$$


where $\rho_{\varepsilon}=\rho+\varepsilon$. Using the fact

$$
\begin{aligned}
\sum_{i=1}^{N} \iint_{Q_{T}} v_{\varepsilon} \frac{\partial a_{i}\left(v_{\varepsilon}\right)}{\partial x_{i}} d x d t & =-\sum_{i=1}^{N} \iint_{Q_{T}} \frac{\partial v_{\varepsilon}}{\partial x_{i}} a_{i}\left(v_{\varepsilon}\right) d x d t \\
& =-\sum_{i=1}^{N} \int_{\Omega} \frac{\partial}{\partial x_{i}} \int_{0}^{v_{\varepsilon}} a_{i}(s) d s d x=0,
\end{aligned}
$$

we have

$$
\frac{\beta}{\beta+1} \int_{\Omega} v_{\varepsilon}^{\beta+1} d x+\delta \iint_{Q_{T}} \rho_{\varepsilon}\left|\nabla v_{\varepsilon}\right|^{p} d x d t \leq c,
$$

and in particular,

$$
\iint_{Q_{T}} \rho\left|\nabla v_{\varepsilon}\right|^{p} d x d t \leq c \iint_{Q_{T}} \rho_{\varepsilon}\left|\nabla v_{\varepsilon}\right|^{p} d x d t \leq c .
$$

For small enough $\lambda>0$, let $\Omega_{\lambda}=\{x \in \Omega: \rho(x)>\lambda\}$. Since $p \geq 2$, by (1.6) and (2.10),

$$
\int_{0}^{T} \int_{\Omega_{\lambda}}\left|\nabla v_{\varepsilon}\right|^{2} d x d t \leq c\left(\int_{0}^{T} \int_{\Omega_{\lambda}}\left|\nabla v_{\varepsilon}\right|^{p} d x d t\right)^{\frac{2}{p}} \leq c(\lambda) .
$$

Multiplying (2.1) with $v_{\varepsilon t}$, and integrating over $\Omega$,

$$
\begin{aligned}
& \beta \int_{\Omega} v_{\varepsilon}^{\beta-1}\left(v_{\varepsilon t}\right)^{2} d x \\
& \quad=\delta \int_{\Omega} \operatorname{div}\left((\rho+\varepsilon)\left|\nabla v_{\varepsilon}\right|^{p-2} \nabla v_{\varepsilon}\right) v_{\varepsilon t} d x+\sum_{i=1}^{N} \int_{\Omega} \frac{\partial a_{i}\left(v_{\varepsilon}\right)}{\partial x_{i}} v_{\varepsilon t} d x \\
& \delta \int_{\Omega} \operatorname{div}\left((\rho+\varepsilon)\left|\nabla v_{\varepsilon}\right|^{p-2} \nabla v_{\varepsilon}\right) v_{\varepsilon t} d x \\
& \quad=-\delta \int_{\Omega}(\rho+\varepsilon)\left|\nabla v_{\varepsilon}\right|^{p-2} \nabla v_{\varepsilon} \nabla v_{\varepsilon t} d x \\
& \quad=-\frac{\delta}{2} \int_{\Omega}(\rho+\varepsilon)\left|\nabla v_{\varepsilon}\right|^{p-2}\left|\nabla v_{\varepsilon}\right|_{t}^{2} d x \\
& \quad=-\frac{\delta}{2} \int_{\Omega}(\rho+\varepsilon) \frac{d}{d t} \int_{0}^{\left|\nabla v_{\varepsilon}\right|^{2}} s^{\frac{p-2}{2}} d s d x .
\end{aligned}
$$

By the assumption of (1.11),

$$
\begin{aligned}
& \sum_{i=1}^{N}\left|\int_{\Omega} \frac{\partial a_{i}\left(v_{\varepsilon}\right)}{\partial x_{i}} v_{\varepsilon t} d x\right| \\
& \quad \leq \sum_{i=1}^{N} \int_{\Omega}\left|a_{i}^{\prime}\left(v_{\varepsilon}\right)\right|\left|v_{\varepsilon x_{i}}\right|\left|v_{\varepsilon t}\right| d x \\
& \quad \leq \frac{\beta}{2} \int_{\Omega} v_{\varepsilon}^{\beta-1}\left(v_{\varepsilon t}\right)^{2} d x+c \int_{\Omega}\left|v_{\varepsilon}\right|^{2 \alpha-\beta+1}\left|\nabla v_{\varepsilon}\right|^{2} d x .
\end{aligned}
$$


By (2.12)-(2.14), we have

$$
\begin{aligned}
& \frac{\beta}{2} \int_{\Omega} v^{\beta-1}\left(v_{\varepsilon}\right)^{2} d x+\frac{\delta}{2} \int_{\Omega}(\rho+\varepsilon) \frac{d}{d t} \int_{0}^{\left|\nabla v_{\varepsilon}\right|^{2}} s^{\frac{p-2}{2}} d s d x \\
& \quad \leq c \int_{\Omega}\left|v_{\varepsilon}\right|^{2 \alpha-\beta+1}\left|\nabla v_{\varepsilon}\right|^{2} d x \\
& \quad \leq c\left(\int_{\Omega}(\rho+\varepsilon)^{-\frac{2}{p-2}} d x\right)^{\frac{p-2}{p}}\left(\int_{\Omega}(\rho+\varepsilon)\left|\nabla v_{\varepsilon}\right|^{p} d x\right)^{\frac{2}{p}} \\
& \quad \leq c .
\end{aligned}
$$

Here, we have used the assumption $\int_{\Omega} \rho(x)^{-\frac{2}{p-2}} d x<\infty$, which implies $\int_{\Omega}(\rho+\varepsilon)^{-\frac{2}{p-2}} d x<$ $\infty$. Then

$$
\left\|\left(v_{\varepsilon}^{\frac{\beta+1}{2}}\right)_{t}\right\|_{L^{2}\left(Q_{T}\right)}=\frac{\beta+1}{2}\left\|v_{\varepsilon}^{\frac{\beta-1}{2}} v_{\varepsilon t}\right\|_{L^{2}\left(Q_{T}\right)} \leq c
$$

and

$$
\begin{aligned}
\iint_{Q_{T}}\left|v_{\varepsilon}\right|^{2} d x d t & \leq \int_{0}^{T} \int_{\Omega} v_{\varepsilon}^{\beta-1}\left|v_{\varepsilon t}\right|^{2} v_{\varepsilon}^{1-\beta} d x d t \\
& \leq\left\|v_{\varepsilon}\right\|_{L^{\infty}\left(Q_{T}\right)}^{1-\beta} \int_{0}^{T} \int_{\Omega} v_{\varepsilon}^{\beta-1}\left|v_{\varepsilon t}\right|^{2} d x d t \leq c
\end{aligned}
$$

From (2.10), (2.11), and (2.16), one knows that

$$
\iint_{Q_{\lambda T}}\left|v_{\varepsilon}\right|^{2} d x d t \leq c, \quad \iint_{Q_{\lambda T}}\left|\nabla v_{\varepsilon}\right|^{2} d x d t \leq c(\lambda),
$$

where $Q_{\lambda T}=\Omega_{\lambda} \times(0, T)$. Then $v_{\varepsilon} \rightarrow v$ in $L^{2}\left(Q_{\lambda T}\right)$. By the arbitrariness of $\lambda, v_{\varepsilon} \rightarrow v$ a.e. in $Q_{T}$. Thus $a_{i}\left(v_{\varepsilon}\right) \rightarrow a_{i}(v)$ a.e. in $Q_{T}$.

We use (2.10), (2.15), and let $\varepsilon \rightarrow 0$. Similarly as in $[3,20]$, we can prove that

$$
(\rho(x)+\varepsilon)\left|\nabla v_{\varepsilon}\right|^{p-2} \nabla v_{\varepsilon} \rightarrow * \rho(x)|\nabla v|^{p-2} \nabla v, \quad \text { weakly star in } L^{1}\left(0, T ; L^{\frac{p}{p-1}}(\Omega)\right),
$$

so that $a_{i}\left(v_{\varepsilon}\right) \rightarrow a_{i}(v)$ a.e. in $Q_{T}$, and then there is a solution of Eq. (1.7) with the initial value (1.8) in the sense of Definition 1.1.

\section{The uniqueness}

Theorem 3.1 Let $u(x, t)$ and $v(x, t)$ be two weak solutions of Eq. (1.7) with different initial values $u_{0}(x)$ and $v_{0}(x)$, respectively, $0<m \leq\|u\|_{L^{\infty}\left(Q_{T}\right)} \leq M, 0<m \leq\|v\|_{L^{\infty}\left(Q_{T}\right)} \leq M$. Let $p>1, a_{i}(s)$ be a Lipschitz function, and let $\rho(x)$ satisfy (1.6). Then there exists a constant $\alpha_{1} \geq \max \{p, 2,2(p-1)\}$ such that

$$
\begin{aligned}
& \left.\int_{\Omega} \rho^{\frac{\alpha_{1}}{p}}|| u\right|^{\beta-1} u(x, t)-\left.|v|^{\beta-1} v(x, t)\right|^{2} d x \\
& \quad \leq\left.\int_{\Omega} \rho^{\frac{\alpha_{1}}{p}}|| u_{0}\right|^{\beta-1} u_{0}(x)-\left.\left|v_{0}\right|^{\beta-1} v_{0}(x)\right|^{2} d x, \quad \forall t \in[0, T) .
\end{aligned}
$$


Proof Denote $\Omega_{\lambda}=\{x \in \Omega: \rho(x)>\lambda\}$ as before. Let

$$
\xi_{\lambda}=[\rho(x)-\lambda]_{+}^{\frac{\alpha_{1}}{p}}
$$

For any fixed $\tau, s \in[0, T]$, we may choose $\chi_{[\tau, s]}\left(u_{\varepsilon}-v_{\varepsilon}\right) \xi_{\lambda}$ as a test function in (3.1), where $\chi_{[\tau, s]}$ is the characteristic function on $[\tau, s]$, where $u_{\varepsilon}$ and $v_{\varepsilon}$ are the mollified functions of the solutions $u$ and $v$, respectively. Then, denoting $Q_{\tau s}=\Omega \times[\tau, s]$, we have

$$
\begin{aligned}
& \iint_{Q_{\tau s}}\left(u_{\varepsilon}-v_{\varepsilon}\right) \xi_{\lambda} \frac{\partial\left(|u|^{\beta-1} u-|v|^{\beta-1} v\right)}{\partial t} d x d t \\
& \quad=-\delta \iint_{Q_{\tau s}}\left(\rho(x)|\nabla u|^{p-2} \nabla u-|\nabla v|^{p-2} \nabla v\right) \nabla\left[\left(u_{\varepsilon}-v_{\varepsilon}\right) \xi_{\lambda}\right] d x d t \\
& \left.\quad-\sum_{i=1}^{N} \iint_{Q_{\tau s}}\left[a_{i}(u)-a_{i}(v)\right]\left[u_{\varepsilon}-v_{\varepsilon}\right) \xi_{\lambda}\right]_{x_{i}} d x d t
\end{aligned}
$$

For any given small $\lambda>0$, denoting $Q_{T \lambda}=\Omega_{\lambda} \times(0, T)$, since $\rho(x) \in C^{1}(\bar{\Omega})$ and $\rho(x)>0$ when $x \in \Omega$, then $\nabla u \in L^{p}\left(Q_{T \lambda}\right), \nabla v \in L^{p}\left(Q_{T \lambda}\right)$. According to the definition of the mollified functions $u_{\varepsilon}$ and $v_{\varepsilon}$, we have

$$
\begin{aligned}
& u_{\varepsilon} \in L^{\infty}\left(Q_{T}\right), \quad v_{\varepsilon} \in L^{\infty}\left(Q_{T}\right), \\
& \left\|\nabla u_{\varepsilon}\right\|_{p, \Omega_{\lambda}} \leq\|\nabla u\|_{p, \Omega_{\lambda}}, \quad\left\|\nabla v_{\varepsilon}\right\|_{p, \Omega_{\lambda}} \leq\|\nabla v\|_{p, \Omega_{\lambda}} .
\end{aligned}
$$

Since on $\Omega_{\lambda}$, by Young inequality,

$$
\begin{aligned}
& \mid \rho(x)\left(|\nabla u|^{p-2} \nabla u-|\nabla v|^{p-2} \nabla v\right) \nabla\left[\left(u_{\varepsilon}-v_{\varepsilon}\right) \mid\right. \\
& \quad \leq c(\lambda)\left(|\nabla u|^{p}+|\nabla v|^{p}\right)+c\left(\left|\nabla u_{\varepsilon}\right|^{p}+\left|\nabla v_{\varepsilon}\right|^{p}\right) \\
& \quad \leq 2 c(\lambda)\left(|\nabla u|^{p}+|\nabla v|^{p}\right),
\end{aligned}
$$

by (3.2), (3.4) and (3.5), using the Lebesgue dominated convergence theorem, we have

$$
\begin{aligned}
\lim _{\varepsilon \rightarrow 0} \iint_{Q_{\tau s}} \rho(x)\left(|\nabla u|^{p-2} \nabla u-|\nabla v|^{p-2} \nabla v\right) \nabla\left[\left(u_{\varepsilon}-v_{\varepsilon}\right) \xi_{\lambda}\right] d x d t \\
=\iint_{Q_{\tau s}} \rho(x)\left(|\nabla u|^{p-2} \nabla u-|\nabla v|^{p-2} \nabla v\right) \nabla\left[(u-v) \xi_{\lambda}\right] d x d t \\
=\iint_{Q_{\tau s}} \rho(x) \xi_{\lambda}\left(|\nabla u|^{p-2} \nabla u-|\nabla v|^{p-2} \nabla v\right) \nabla(u-v) d x d t \\
\quad+\iint_{Q_{\tau s}} \rho(x)\left(|\nabla u|^{p-2} \nabla u-|\nabla v|^{p-2} \nabla v\right)(u-v) \nabla \xi_{\lambda} d x d t .
\end{aligned}
$$

The first term on the right-hand side of (3.6) satisfies

$$
\iint_{Q_{\tau s}} \rho(x) \xi_{\lambda}\left(|\nabla u|^{p-2} \nabla u-|\nabla v|^{p-2} \nabla v\right) \nabla(u-v) d x d t \geq 0
$$


The last term on the right-hand side of (3.6) can be bounded as follows:

$$
\begin{aligned}
& \left|\iint_{Q_{\tau s}}(u-v) \rho(x)\left(|\nabla u|^{p-2} \nabla u-|\nabla v|^{p-2} \nabla v\right) \nabla \xi_{\lambda} d x d t\right| \\
& \quad \leq c\left(\int_{\tau}^{s} \int_{\Omega_{\lambda}} \rho(x)\left(|\nabla u|^{p}+|\nabla v|^{p}\right) d x d t\right)^{\frac{p-1}{p}} \cdot\left(\int_{\tau}^{s} \int_{\Omega_{\lambda}} \rho(x)\left|\nabla \xi_{\lambda}\right|^{p}|u-v|^{p} d x d t\right)^{\frac{1}{p}} \\
& \quad \leq c\left(\int_{\tau}^{s} \int_{\Omega} \rho(x)\left(|\nabla u|^{p}+|\nabla v|^{p}\right) d x d t\right)^{\frac{p-1}{p}} \\
& \quad \cdot\left(\int_{\tau}^{s} \int_{\Omega_{\lambda}} \rho(x)[\rho(x)-\lambda]^{p\left(\frac{\alpha_{1}}{p}-1\right)}|\nabla \rho|^{p}|u-v|^{p} d x d t\right)^{\frac{1}{p}} \\
& \quad \leq c\left(\int_{\tau}^{s} \int_{\Omega_{\lambda}} \rho(x)[\rho(x)-\lambda]^{p\left(\frac{\alpha_{1}}{p}-1\right)}|u-v|^{p} d x d t\right)^{\frac{1}{p}} .
\end{aligned}
$$

Here, we have used the fact that $|\nabla \rho(x)| \leq c$. Then,

$$
\begin{aligned}
& \lim _{\lambda \rightarrow 0}\left|\iint_{Q_{\tau s}}(u-v) \rho(x)\left(|\nabla u|^{p-2} \nabla u-|\nabla v|^{p-2} \nabla v\right) \nabla \xi_{\lambda} d x d t\right| \\
& \quad \leq \lim _{\lambda \rightarrow 0} c\left(\int_{\tau}^{s} \int_{\Omega_{\lambda}} \rho(x)(\rho-\lambda)^{p\left(\frac{\alpha_{1}}{p}-1\right)}|u-v|^{p} d x d t\right)^{\frac{1}{p}} \\
& \quad \leq c\left(\int_{\tau}^{s} \int_{\Omega} \rho^{1+p\left(\frac{\alpha_{1}}{p}-1\right)}|u-v|^{p} d x d t\right)^{\frac{1}{p}} .
\end{aligned}
$$

If $p \geq 2$, clearly, since $u, v \in L^{\infty},|u-v| \leq c$, and we have

$$
\left(\int_{\tau}^{s} \int_{\Omega} \rho^{1+p\left(\frac{\alpha_{1}}{p}-1\right)}|u-v|^{p} d x d t\right)^{\frac{1}{p}} \leq c\left(\int_{\tau}^{s} \int_{\Omega} \rho^{\frac{\alpha_{1}}{p}}|u-v|^{2} d x d t\right)^{\frac{1}{p}}
$$

if $1<p<2$, for $\alpha_{1} \geq 2(p-1)$, using the Hölder inequality, we have

$$
\begin{aligned}
& \left(\int_{\tau}^{s} \int_{\Omega} \rho^{1+p\left(\frac{\alpha_{1}}{p}-1\right)}|u-v|^{p} d x d t\right)^{\frac{1}{p}} \\
& \quad \leq\left(\int_{\tau}^{s} \int_{\Omega}\left[\rho^{1+p\left(\frac{\alpha_{1}}{p}-1\right)-\frac{\alpha_{1}}{2}}\right]^{\frac{2}{2-p}}\right)^{\frac{2-p}{2} \frac{1}{p}}\left(\int_{\tau}^{s} \int_{\Omega} \rho^{\frac{\alpha_{1}}{p}}|u-v|^{2} d x d t\right)^{\frac{1}{2}} \\
& \quad \leq c\left(\int_{\tau}^{s} \int_{\Omega} \rho^{\frac{\alpha_{1}}{p}}|u-v|^{2} d x d t\right)^{\frac{1}{2}} .
\end{aligned}
$$

Meanwhile, by the Lebesgue dominated convergence theorem,

$$
\begin{array}{r}
\left.\lim _{\varepsilon \rightarrow 0} \iint_{Q_{\tau s}}\left[a_{i}(u)-a_{i}(v)\right]\left[u_{\varepsilon}-v_{\varepsilon}\right) \xi_{\lambda}\right]_{x_{i}} d x d t \\
\quad=\iint_{Q_{\tau s}}\left[a_{i}(u)-a_{i}(v)\right]\left[(u-v) \xi_{\lambda}\right]_{x_{i}} d x d t
\end{array}
$$




$$
\begin{aligned}
& =\iint_{Q_{\tau s}}\left[a_{i}(u)-a_{i}(v)\right](u-v) \xi_{\lambda x_{i}} d x d t \\
& \quad+\iint_{Q_{s}}\left[a_{i}(u)-a_{i}(v)\right](u-v)_{x_{i}} \xi_{\lambda} d x d t .
\end{aligned}
$$

Due to the fact $|\nabla \rho| \leq c, \alpha_{1} \geq p$, we have

$$
\begin{aligned}
& \lim _{\lambda \rightarrow 0} \iint_{Q_{\tau s}}\left[a_{i}(u)-a_{i}(v)\right](u-v) \xi_{\lambda x_{i}} d x d t \\
& \quad \leq c \lim _{\lambda \rightarrow 0} \int_{\tau}^{s} \int_{\Omega_{\lambda}}\left[a_{i}(u)-a_{i}(v)\right](u-v)[\rho-\lambda]^{\frac{\alpha_{1}}{p}-1}\left|\rho_{x_{i}}\right| d x \\
& \quad \leq c\left(\int_{\tau}^{s} \int_{\Omega} \rho^{\frac{\alpha_{1}}{p}}|u-v|^{2} d x\right)^{\frac{1}{2}}
\end{aligned}
$$

and

$$
\begin{aligned}
\lim _{\lambda \rightarrow 0} \iint_{Q_{\tau s}}\left[a_{i}(u)-a_{i}(v)\right](u-v)_{x_{i}} \xi_{\lambda} d x d t \\
=\lim _{\lambda \rightarrow 0} \iint_{Q_{\tau s}}\left[a_{i}(u)-a_{i}(v)\right](u-v)_{x_{i}}[\rho-\lambda]_{+}^{\frac{\alpha_{1}}{p}} d x d t \\
\leq\left(\int_{\tau}^{s} \int_{\Omega}\left(\rho^{\frac{\alpha_{1}-1}{p}}\right)^{p^{\prime}}\left|a_{i}(u)-a_{i}(v)\right|^{p^{\prime}} d x d t\right)^{\frac{1}{p^{\prime}}} \\
\quad \cdot\left(\int_{\tau}^{s} \int_{\Omega} \rho(x)\left(|\nabla u|^{p}+|\nabla v|^{p}\right) d x d t\right)^{\frac{1}{p}} \\
\leq c\left(\int_{\tau}^{s} \int_{\Omega} \rho^{\frac{\alpha_{1}-1}{p-1}}\left|a_{i}(u)-a_{i}(v)\right|^{p^{\prime}} d x d t\right)^{\frac{1}{p^{\prime}}} .
\end{aligned}
$$

If $1<p<2$, then $p^{\prime}>2$, and if $\alpha_{1} \geq p$, when $\rho<1$, then $\rho^{\frac{\alpha_{1}-1}{p-1}} \leq \rho^{\frac{\alpha_{1}}{p}}$. When $1 \leq \rho \leq D=$ $\sup _{x \in \bar{\Omega}} \rho(x)$, it is obvious that

$$
\rho^{\frac{\alpha_{1}-1}{p-1}-\frac{\alpha_{1}}{p}}=\rho^{\frac{\alpha_{1}-p}{p(p-1)}} \leq D^{\frac{\alpha_{1}-p}{p(p-1)}} \leq c .
$$

Thus, $\rho^{\frac{\alpha_{1}-1}{p-1}} \leq c \rho^{\frac{\alpha_{1}}{p}}$ is always true, and then we have

$$
\left(\int_{\tau}^{s} \int_{\Omega} \rho^{\frac{\alpha_{1}-1}{p-1}}\left|a_{i}(u)-a_{i}(v)\right|^{p^{\prime}} d x d t\right)^{\frac{1}{p^{\prime}}} \leq c\left(\int_{\tau}^{s} \int_{\Omega} \rho^{\frac{\alpha_{1}}{p}}|u-v|^{2} d x d t\right)^{\frac{1}{p^{\prime}}}
$$

If $p \geq 2$, then $p^{\prime}<2$, and for $\alpha_{1} \geq 2$, by the Hölder inequality,

$$
\begin{aligned}
& \left(\int_{\tau}^{s} \int_{\Omega} \rho^{\frac{\alpha_{1}-1}{p-1}}\left|a_{i}(u)-a_{i}(v)\right|^{p^{\prime}} d x d t\right)^{\frac{1}{p^{\prime}}} \\
& \quad \leq c\left(\int_{\tau}^{s} \int_{\Omega}\left[\rho^{\frac{\alpha_{1}-1}{p-1}-\frac{\alpha_{1}}{2(p-1)}}\right]^{\frac{2}{2-p^{\prime}}} d x d t\right)^{\frac{2-p^{\prime}}{2} \frac{1}{p^{\prime}}}\left(\int_{\tau}^{s} \int_{\Omega} \rho^{\frac{\alpha_{1}}{p}}|u-v|^{2} d x d t\right)^{\frac{1}{2}} .
\end{aligned}
$$


By (3.6)-(3.14), after letting $\varepsilon \rightarrow 0$, we let $\lambda \rightarrow 0$ in (3.3). Then

$$
\begin{aligned}
& \iint_{Q_{\tau s}} \rho^{\frac{\alpha_{1}}{p}}(u-v) \frac{\partial\left(|u|^{\beta-1} u-|v|^{\beta-1} v\right)}{\partial t} d x d t \\
& \quad \leq c\left(\int_{0}^{s} \int_{\Omega} \rho^{\frac{\alpha_{1}}{p}}|u(x, t)-v(x, t)|^{2} d x d t\right)^{q},
\end{aligned}
$$

where $q<1$. Now,

$$
\begin{aligned}
& \iint_{Q_{\tau s}} \rho^{\frac{\alpha_{1}}{p}}(u-v) \frac{\partial\left(|u|^{\beta-1} u-|v|^{\beta-1} v\right)}{\partial t} d x d t \\
& \quad=\iint_{Q_{\tau s}} \rho^{\frac{\alpha_{1}}{p}} \frac{u-v}{|u|^{\beta-1} u-|v|^{\beta-1} v}\left(|u|^{\beta-1} u-|v|^{\beta-1} v\right) \frac{\partial\left(|u|^{\beta-1} u-|v|^{\beta-1} v\right)}{\partial t} d x d t \\
& \quad=\frac{1}{2} \iint_{Q_{\tau s}} \frac{\rho^{\frac{\alpha_{1}}{p}}}{\beta|\zeta|^{\beta-1}} \frac{\partial\left(|u|^{\beta-1} u-|v|^{\beta-1} v\right)^{2}}{\partial t} d x d t
\end{aligned}
$$

where $\zeta \in(v, u)$.

If for any $s \geq \tau$

$$
\frac{d}{d t}\left\|\rho^{\frac{\alpha_{1}}{2 p}}\left(|u|^{\beta-1} u-|v|^{\beta-1} v\right)\right\|_{L^{2}(\Omega)} \leq 0, \quad t \in[\tau, s]
$$

is true, then

$$
\left.\int_{\Omega} \rho^{\frac{\alpha_{1}}{p}}|| u\right|^{\beta-1} u(x, s)-\left.|v|^{\beta-1} v(x, s)\right|^{2} d x \leq\left.\int_{\Omega} \rho^{\frac{\alpha_{1}}{p}}|| u\right|^{\beta-1} u(x, \tau)-\left.|v|^{\beta-1} v(x, \tau)\right|^{2} d x
$$

clearly holds.

If there is an $s_{0} \geq \tau$ such that

$$
\frac{d}{d t}\left\|\rho^{\frac{\alpha_{1}}{2 p}}\left(|u|^{\beta-1} u-|v|^{\beta-1} v\right)\right\|_{L^{2}(\Omega)}>0, \quad t \in\left[\tau, s_{0}\right]
$$

then by (3.16)

$$
\begin{aligned}
& \iint_{Q_{\tau s_{0}}} \rho^{\frac{\alpha_{1}}{p}}(u-v) \frac{\partial\left(|u|^{\beta-1} u-|v|^{\beta-1} v\right)}{\partial t} d x d t \\
& \quad=\frac{1}{2} \iint_{Q_{\tau s_{0}}} \frac{\rho^{\frac{\alpha_{1}}{p}}}{\beta|\zeta|^{\beta-1}} \frac{\partial\left(|u|^{\beta-1} u-|v|^{\beta-1} v\right)^{2}}{\partial t} d x d t \\
& \quad \geq \frac{1}{2 \beta M^{\beta-1}} \iint_{Q_{\tau s_{0}}} \rho^{\frac{\alpha_{1}}{p}} \frac{\partial\left(|u|^{\beta-1} u-|v|^{\beta-1} v\right)^{2}}{\partial t} d x d t
\end{aligned}
$$

where $\zeta \in(v, u), M=\max \left\{\|u\|_{L^{\infty}\left(Q_{T}\right)},\|v\|_{L^{\infty}\left(Q_{T}\right)}\right\}$.

By (3.15), (3.16), and (3.19),

$$
\begin{aligned}
& \int_{\tau}^{s_{0}} \frac{\partial}{\partial t} \int_{\Omega} \rho^{\frac{\alpha_{1}}{p}}\left(|u|^{\beta-1} u-|v|^{\beta-1} v\right)^{2} d x d t \\
& \quad \leq 2 c \beta M^{\beta-1}\left(\int_{\tau}^{s_{0}} \int_{\Omega} \rho^{\frac{\alpha_{1}}{p}}|u(x, t)-v(x, t)|^{2} d x d t\right)^{q} .
\end{aligned}
$$


Then

$$
\begin{aligned}
& \left.\int_{\Omega} \rho^{\frac{\alpha_{1}}{p}}|| u\right|^{\beta-1} u\left(x, s_{0}\right)-\left.|v|^{\beta-1} v\left(x, s_{0}\right)\right|^{2} d x \\
& \quad \leq\left.\int_{\Omega} \rho^{\frac{\alpha_{1}}{p}}|| u\right|^{\beta-1} u(x, \tau)-\left.|v|^{\beta-1} v(x, \tau)\right|^{2} d x \\
& \quad+2 c \beta M^{\beta-1}\left(\int_{\tau}^{s_{0}} \int_{\Omega} \rho^{\frac{\alpha_{1}}{p}}|u(x, t)-v(x, t)|^{2} d x d t\right)^{q} .
\end{aligned}
$$

Since

$$
\left.|| u\right|^{\beta-1} u-\left.|v|^{\beta-1} v|=| \frac{|u|^{\beta-1} u-|v|^{\beta-1} v}{u-v}(u-v)|=\beta| \zeta\right|^{\beta-1}|u-v|,
$$

by (3.20), we have

$$
\begin{aligned}
& \left.\int_{\Omega} \rho^{\frac{\alpha_{1}}{p}}|| u\right|^{\beta-1} u\left(x, s_{0}\right)-\left.|v|^{\beta-1} v\left(x, s_{0}\right)\right|^{2} d x \\
& \leq\left.\int_{\Omega} \rho^{\frac{\alpha_{1}}{p}}|| u\right|^{\beta-1} u(x, \tau)-\left.|v|^{\beta-1} v(x, \tau)\right|^{2} d x \\
& \quad+\frac{2 c M^{\beta-1}}{\beta m^{2(\beta-1)}}\left(\left.\int_{\tau}^{s_{0}} \int_{\Omega} \rho^{\frac{\alpha_{1}}{p}}|| u\right|^{\beta-1} u(x, t)-\left.|v|^{\beta-1} v(x, t)\right|^{2} d x d t\right)^{q} .
\end{aligned}
$$

Here $m=\min \left\{\|u\|_{L^{\infty}\left(Q_{T}\right)},\|v\|_{L^{\infty}\left(Q_{T}\right)}\right\}$. Inequality (3.21) implies

$$
\begin{aligned}
& \left.\int_{\Omega} \rho^{\frac{\alpha_{1}}{p}}|| u\right|^{\beta-1} u\left(x, s_{0}\right)-\left.|v|^{\beta-1} v\left(x, s_{0}\right)\right|^{2} d x \\
& \quad \leq\left.\int_{\Omega} \rho^{\frac{\alpha_{1}}{p}}|| u\right|^{\beta-1} u(x, \tau)-\left.|v|^{\beta-1} v(x, \tau)\right|^{2} d x, \quad t \in\left[\tau, s_{0}\right] .
\end{aligned}
$$

This inequality contradicts assumption (3.18).

Thus, for any $s, \tau \in[0, T)$, (3.17) is always true. By the arbitrariness of $\tau$, we have

$$
\left.\int_{\Omega} \rho^{\frac{\alpha_{1}}{p}}|| u\right|^{\beta-1} u(x, s)-\left.|v|^{\beta-1} v(x, s)\right|^{2} d x \leq\left.\int_{\Omega} \rho^{\frac{\alpha_{1}}{p}}|| u_{0}\right|^{\beta-1} u_{0}(x)-\left.\left|v_{0}\right|^{\beta-1} v_{0}(x)\right|^{2} d x .
$$

The proof is complete.

Since $\rho$ satisfies (1.6), from (3.1), we can deduce Theorem 1.3.

\section{The proof of Theorem 1.4}

Proof For any given positive integer $n$, let $g_{n}(s)$ be an odd function, and

$$
g_{n}(s)= \begin{cases}1, & s>\frac{1}{n} \\ n^{2} s^{2} e^{1-n^{2} s^{2}}, & 0 \leq s \leq \frac{1}{n}\end{cases}
$$

Clearly,

$$
\lim _{n \rightarrow \infty} g_{n}(s)=\operatorname{sgn}(s), \quad s \in(-\infty,+\infty)
$$


and

$$
0 \leq g_{n}^{\prime}(s) \leq \frac{c}{s}, \quad 0<s<\frac{1}{n},
$$

where $c$ is independent of $n$.

Let $u_{\varepsilon}$ and $v_{\varepsilon}$ be the asymptotic solutions of $u$ and $v$, respectively. They satisfy the asymptotic problem (2.1)-(2.3). Since the weak solution of Eq. (1.7) with the initial value (1.8) is unique, we have

$$
\begin{array}{ll}
\lim _{\varepsilon \rightarrow 0} u_{\varepsilon}=u, \quad \lim _{\varepsilon \rightarrow 0} v_{\varepsilon}=v, & \text { a.e. }(x, t) \in Q_{T}, \\
\rho^{\frac{1}{p}}(x) \nabla u_{\varepsilon} \rightarrow \rho^{\frac{1}{p}}(x) \nabla u, & \rho^{\frac{1}{p}}(x) \nabla v_{\varepsilon} \rightarrow \rho^{\frac{1}{p}}(x) \nabla v, \quad \text { in } L^{p}(\Omega) .
\end{array}
$$

We can choose $\chi_{[\tau, s]} g_{n}\left(u_{\varepsilon}-v_{\varepsilon}\right)$ as the test function. Then

$$
\begin{aligned}
& \int_{s}^{t} \int_{\Omega} g_{n}\left(u_{\varepsilon}-v_{\varepsilon}\right) \frac{\partial\left(|u|^{\beta-1} u-|v|^{\beta-1} v\right)}{\partial t} d x d t \\
& \quad+\delta \int_{s}^{t} \int_{\Omega} \rho(x)\left(|\nabla u|^{p-2} \nabla u-|\nabla v|^{p-2} \nabla v\right) \cdot \nabla\left(u_{\varepsilon}-v_{\varepsilon}\right) g_{n}^{\prime} d x d t \\
& \quad+\sum_{i=1}^{N} \int_{s}^{t} \int_{\Omega}\left[a_{i}(u)-a_{i}(v)\right] \cdot\left(u_{\varepsilon}-v_{\varepsilon}\right)_{x_{i}} g_{n}^{\prime}\left(u_{\varepsilon}-v_{\varepsilon}\right) d x d t \\
& =0 .
\end{aligned}
$$

At first, by (4.4), we have

$$
\lim _{\varepsilon \rightarrow 0} \int_{\Omega}\left[\rho^{\frac{p-1}{p}}\left(|\nabla u|^{p-2} \nabla u-|\nabla v|^{p-2} \nabla v\right)\right] \rho^{\frac{1}{p}}\left[\nabla\left(u_{\varepsilon}-v_{\varepsilon}\right)-\nabla(u-v)\right] d x d t=0 .
$$

Secondly, we have

$$
\begin{aligned}
\lim _{\varepsilon \rightarrow 0} & \int_{\Omega} \rho^{\frac{p-1}{p}}\left(|\nabla u|^{p-2} \nabla u-|\nabla v|^{p-2} \nabla v\right) \rho^{\frac{1}{p}}\left[\nabla\left(u_{\varepsilon}-v_{\varepsilon}\right)-\nabla(u-v)\right] g_{n}^{\prime}\left(u_{\varepsilon}-v_{\varepsilon}\right) d x d t \\
= & \lim _{\varepsilon \rightarrow 0} \int_{\Omega} \rho^{\frac{p-1}{p}}\left(|\nabla u|^{p-2} \nabla u-|\nabla v|^{p-2} \nabla v\right) \rho^{\frac{1}{p}}\left[\nabla\left(u_{\varepsilon}-v_{\varepsilon}\right)-\nabla(u-v)\right]_{n}^{\prime}(u-v) d x d t \\
& \quad+\lim _{\varepsilon \rightarrow 0} \int_{\Omega} \rho^{\frac{p-1}{p}}(x)\left(|\nabla u|^{p-2} \nabla u-|\nabla v|^{p-2} \nabla v\right) \\
& \cdot \rho^{\frac{1}{p}}\left[\nabla\left(u_{\varepsilon}-v_{\varepsilon}\right)-\nabla(u-v)\right]\left[g_{n}^{\prime}\left(u_{\varepsilon}-v_{\varepsilon}\right)-g_{n}^{\prime}(u-v)\right] d x d t \\
= & \lim _{\varepsilon \rightarrow 0} \int_{\Omega} \rho^{\frac{p-1}{p}}\left(|\nabla u|^{p-2} \nabla u-|\nabla v|^{p-2} \nabla v\right) \rho^{\frac{1}{p}}\left[\nabla\left(u_{\varepsilon}-v_{\varepsilon}\right)-\nabla(u-v)\right] g_{n}^{\prime}(u-v) d x d t \\
= & 0 .
\end{aligned}
$$


Here, we have used two facts. The first one is, by (4.6),

$$
\begin{aligned}
& \lim _{\varepsilon \rightarrow 0} \int_{\Omega} \rho^{\frac{p-1}{p}}\left(|\nabla u|^{p-2} \nabla u-|\nabla v|^{p-2} \nabla v\right) \rho^{\frac{1}{p}}\left[\nabla\left(u_{\varepsilon}-v_{\varepsilon}\right)-\nabla(u-v)\right] g_{n}^{\prime}(u-v) d x d t \\
& \quad=g_{n}^{\prime}(\zeta) \lim _{\varepsilon \rightarrow 0} \int_{\Omega} \rho^{\frac{p-1}{p}}(x)\left(|\nabla u|^{p-2} \nabla u-|\nabla v|^{p-2} \nabla v\right) \rho^{\frac{1}{p}}\left[\nabla\left(u_{\varepsilon}-v_{\varepsilon}\right)-\nabla(u-v)\right] d x d t \\
& \quad=0 .
\end{aligned}
$$

The second one is, since $\left(u_{\varepsilon}-v_{\varepsilon}\right) \rightarrow(u-v)$, a.e. in $\Omega$,

$$
\left|\left[g_{n}^{\prime}\left(u_{\varepsilon}-v_{\varepsilon}\right)-g_{n}^{\prime}(u-v)\right]\right| \leq c(n),
$$

using (4.6),

$$
\begin{aligned}
& \lim _{\varepsilon \rightarrow 0} \int_{\Omega} \rho^{\frac{p-1}{p}}(x)\left(|\nabla u|^{p-2} \nabla u-|\nabla v|^{p-2} \nabla v\right) \\
& \quad \times \rho^{\frac{1}{p}}\left[\nabla\left(u_{\varepsilon}-v_{\varepsilon}\right)-\nabla(u-v)\right]\left[g_{n}^{\prime}\left(u_{\varepsilon}-v_{\varepsilon}\right)-g_{n}^{\prime}(u-v)\right] d x d t \\
& \quad=0 .
\end{aligned}
$$

Thirdly, we have

$$
\begin{aligned}
& \lim _{\varepsilon \rightarrow 0} \int_{\Omega} \rho(x)\left(|\nabla u|^{p-2} \nabla u-|\nabla v|^{p-2} \nabla v\right) \nabla\left(u_{\varepsilon}-v_{\varepsilon}\right) g_{n}^{\prime}\left(u_{\varepsilon}-v_{\varepsilon}\right) d x d t \\
& \quad=\iint_{Q_{\tau s}} \rho(x)\left(|\nabla u|^{p-2} \nabla u-|\nabla v|^{p-2} \nabla v\right) \nabla(u-v) g_{n}^{\prime}(u-v) d x d t \geq 0 .
\end{aligned}
$$

Moreover, since

$$
\begin{aligned}
& \int_{\Omega}\left|\rho^{\frac{1}{p}}\left(u_{\varepsilon}-v_{\varepsilon}\right)_{x_{i}} g_{n}^{\prime}\left(u_{\varepsilon}-v_{\varepsilon}\right)\right|^{p} d x \\
& \quad \leq c(n) \int_{\Omega}\left|\rho^{\frac{1}{p}}\left(u_{\varepsilon}-v_{\varepsilon}\right)_{x_{i}}\right|^{p} d x \leq c(n),
\end{aligned}
$$

we have

$$
\rho^{\frac{1}{p}} g_{n}^{\prime}\left(u_{\varepsilon}-v_{\varepsilon}\right)\left(u_{\varepsilon}-v_{\varepsilon}\right)_{x_{i}} \rightarrow \rho^{\frac{1}{p}}(u-v)_{x_{i}} g_{n}^{\prime}(u-v), \quad \text { in } L^{p}(\Omega) .
$$

Then

$$
\begin{gathered}
\lim _{\varepsilon \rightarrow 0} \int_{\Omega}\left[a_{i}(u)-a_{i}(v)\right]\left(u_{\varepsilon}-v_{\varepsilon}\right)_{x_{i}} g_{n}^{\prime}\left(u_{\varepsilon}-v_{\varepsilon}\right) d x \\
=\int_{\Omega}\left[a_{i}(u)-a_{i}(v)\right] \cdot(u-v)_{x_{i}} g_{n}^{\prime}(u-v) d x .
\end{gathered}
$$

Based on it, we are able to prove that

$$
\lim _{n \rightarrow \infty} \int_{\Omega}\left[a_{i}(u)-a_{i}(v)\right] \cdot(u-v)_{x_{i}} g_{n}^{\prime}(u-v) d x=0
$$


In details, the limitation (4.9) is established by the following calculations:

$$
\begin{aligned}
& \left|\int_{\left\{x \in \Omega:|u-v|<\frac{1}{n}\right\}}\left[a_{i}(u)-a_{i}(v)\right] g_{n}^{\prime}(u-v)(u-v)_{x_{i}} d x\right| \\
& \leq c \int_{\left\{x \in \Omega:|u-v|<\frac{1}{n}\right\}}\left|\frac{a_{i}(u)-a_{i}(v)}{u-v}\right|\left|(u-v)_{x_{i}}\right| d x \\
& \leq c\left[\int_{\left\{x \in \Omega:|u-v|<\frac{1}{n}\right\}}\left|\rho^{-\frac{1}{p}} \frac{a_{i}(u)-a_{i}(v)}{u-v}\right|^{\frac{p}{p-1}} d x\right]^{\frac{p-1}{p}} \\
& \cdot\left[\int_{\left\{x \in \Omega:|u-v|<\frac{1}{n}\right\}}\left|\rho^{\frac{1}{p}} \nabla(u-v)\right|^{p} d x\right]^{\frac{1}{p}} .
\end{aligned}
$$

Due to (1.12),

$$
\begin{aligned}
& \int_{\left\{x \in \Omega:|u-v|<\frac{1}{n}\right\}}\left|\rho^{-\frac{1}{p}} \frac{a_{i}(u)-a_{i}(v)}{u-v}\right|^{\frac{p}{p-1}} d x \\
& \quad \leq \int_{\Omega} \rho^{-\frac{1}{p-1}}\left|a_{i}^{\prime}(\xi)\right|^{\frac{p}{p-1}} d x \leq c \int_{\Omega} \rho^{-\frac{1}{p-1}} d x \\
& \quad \leq c,
\end{aligned}
$$

where $\xi \in(v, u)$.

In (4.10), let $n \rightarrow \infty$. If $\{x \in \Omega:|u-v|=0\}$ is a set of zero measure, by (4.11), then

$$
\lim _{n \rightarrow \infty} \int_{\left\{x \in \Omega:|u-v|<\frac{1}{n}\right\}}\left|\rho^{\frac{-1}{p-1}} a_{i}^{\prime}(\xi)\right| d x=\int_{\{x \in \Omega:|u-v|=0\}}\left|\rho^{\frac{-1}{p-1}} a_{i}^{\prime}(\xi)\right| d x=0
$$

If the set $\{x \in \Omega:|u-v|=0\}$ has positive measure, then

$$
\lim _{n \rightarrow \infty} \int_{\left\{x \in \Omega:|u-v|<\frac{1}{n}\right\}} \rho|\nabla(u-v)|^{p} d x=\int_{\{x \in \Omega:|u-v|=0\}} \rho|\nabla(u-v)|^{p} d x=0 .
$$

Therefore, in both cases, the right-hand side of inequality (4.10) goes to 0 as $n \rightarrow \infty$. So (4.9) is true.

Now, letting $n \rightarrow \infty$ in (4.5),

$$
\begin{aligned}
& \lim _{n \rightarrow \infty} \lim _{\varepsilon \rightarrow 0} \int_{\Omega} g_{n}\left(u_{\varepsilon}-v_{\varepsilon}\right) \frac{\partial\left(|u|^{\beta-1} u-|v|^{\beta-1} v\right)}{\partial t} d x \\
& \quad=\lim _{n \rightarrow \infty} \int_{\Omega} g_{n}(u-v) \frac{\partial\left(|u|^{\beta-1} u-|v|^{\beta-1} v\right)}{\partial t} d x \\
& =\int_{\Omega} \operatorname{sgn}(u-v) \frac{\partial\left(|u|^{\beta-1} u-|v|^{\beta-1} v\right)}{\partial t} d x \\
& =\int_{\Omega} \operatorname{sgn}\left(|u|^{\beta-1} u-|v|^{\beta-1} v\right) \frac{\partial\left(|u|^{\beta-1} u-|v|^{\beta-1} v\right)}{\partial t} d x \\
& =\frac{d}{d t}\left\||u|^{\beta-1} u-|v|^{\beta-1} v\right\|_{1} .
\end{aligned}
$$


Then, by (4.6), (4.7), (4.8), (4.9) and (4.12), we have

$$
\frac{d}{d t}\left\||u|^{\beta-1} u-|v|^{\beta-1} v\right\|_{1} \leq 0
$$

It implies that

$$
\left.\int_{\Omega}|| u\right|^{\beta-1} u(x, t)-|v|^{\beta-1} v(x, t)\left|d x \leq \int_{\Omega}\right|\left|u_{0}\right|^{\beta-1} u_{0}-\left|v_{0}\right|^{\beta-1} v_{0} \mid d x, \quad \forall t \in[0, T) .
$$

Theorem 1.4 is proved.

\section{Conclusions}

The equation considered in this paper comes from many reaction-diffusion problems. If the diffusion coefficient not only depends on the unknown solution $u$, but also on the spatial variable $x$, the degeneracy of the equation becomes more complicated. If the diffusion coefficient is degenerate on the boundary, the usual Dirichlet boundary value condition seems redundant completely. The uniqueness of the weak solution is proved without any boundary value conditions. Based on this fact, the stability of weak solutions can also be proved without any boundary value conditions.

\section{Acknowledgements}

The author would like to thank SpringerOpen Accounts Team for its kindness in giving me a discount on the paper charge if my paper gets accepted.

Funding

The paper is supported by Natural Science Foundation of Fujian province, supported by Science Foundation of Xiamen University of Technology, China.

Availability of data and materials

Not applicable.

\section{Competing interests}

The author declares that he has no competing interests.

\section{Authors' contributions}

The author read and approved the final manuscript.

\section{Publisher's Note}

Springer Nature remains neutral with regard to jurisdictional claims in published maps and institutional affiliations.

Received: 13 March 2018 Accepted: 21 January 2019 Published online: 29 January 2019

\section{References}

1. Nakao, M.: $L^{p}$ estimates of solutions of some nonlinear degenerate diffusion equation. J. Math. Soc. Jpn. 37, 41-63 (1985)

2. Wu, Z., Zhao, J., Yin, J., Li, H.: Nonlinear Diffusion Equations. Word Scientific Publishing, Singapore (2001)

3. Zhao, J., Yuan, H.: The Cauchy problem of some doubly nonlinear degenerate parabolic equations. Chin. Ann. Math., Ser. A 16(2), 179-194 (1995) (in Chinese)

4. Benedikt, J., Bobkov, V.E., Girg, P., Kotrla, L., Takac, P.: Nonuniqueness of solutions of initial-value problems for parabolic p-Laplacian. Electron. J. Differ. Equ. 2015, 38 (2015)

5. Benedikt, J., Girg, P., Kotrla, L., Kotrla, L., Takac, P.: Nonuniqueness and multi-bump solutions in parabolic problems with the $p$-Laplacian. J. Differ. Equ. 260, 991-1009 (2016)

6. Zhan, H.: On a parabolic equation related to the $p$-Laplacian. Bound. Value Probl. 2016, 78 (2016)

7. Zhan, H.: The degeneracy on the boundary of an equation related to the $p$-Laplacian. J. Inequal. Appl. 2018, 7 (2018)

8. Zhan, H.: The stability of the solutions of an equation related to the $p$-Laplacian with degeneracy on the boundary. Bound. Value Probl. 2016, 178 (2016)

9. Wu, Z., Zhao, J.: The first boundary value problem for quasilinear degenerate parabolic equations of second order in several variables. Chin. Ann. Math. 4B(1), 57-76 (1983)

10. $\mathrm{Wu}, \mathrm{Z}$., Zhao, J.: Some general results on the first boundary value problem for quasilinear degenerate parabolic equations. Chin. Ann. Math. 4B(3), 319-328 (1983) 
11. Li, Y., Wang, Q.: Homogeneous Dirichlet problems for quasilinear anisotropic degenerate parabolic-hyperbolic equations. J. Differ. Equ. 252, 4719-4741 (2012)

12. Lions, P.L., Perthame, B., Tadmor, E.: A kinetic formation of multidimensional conservation laws and related equations. J. Am. Math. Soc. 7, 169-191 (1994)

13. Kobayasi, K., Ohwa, H.: Uniqueness and existence for anisotropic degenerate parabolic equations with boundary conditions on a bounded rectangle. J. Differ. Equ. 252, 137-167 (2012)

14. Escobedo, M., Vazquez, J.L., Zuazua, E.: Entropy solutions for diffusion-convection equations with partial diffusivity. Trans. Am. Math. Soc. 343, 829-842 (1994)

15. Guarguaglini, F.R., Milišić, V., Terracina, A.: A discrete BGK approximation for strongly degenerate parabolic problems with boundary conditions. J. Differ. Equ. 202, 183-207 (2004)

16. Yin, J., Wang, C.: Properties of the boundary flux of a singular diffusion process. Chin. Ann. Math. 25B(2), 175-182 (2004)

17. Zhan, H., Yuan, H.: A diffusion convection equation with degeneracy on the boundary. J. Jilin Univ. Sci. Ed. 53(3), 353-358 (2015) (in Chinese)

18. Zhan, H.: The boundary value condition of an evolutionary $p(x)$-Laplacian equation. Bound. Value Probl. 2015, 112 (2015). https://doi.org/10.1186/s13661-015-0377-6

19. Zhan, H.: The solutions of a hyperbolic-parabolic mixed type equation on half-space domain. J. Differ. Equ. 259, 1449-1481 (2015)

20. Chen, S., Wang, Y.: Global existence and $L^{\infty}$ estimates of solution for doubly degenerate parabolic equation. Acta Math. Sin., Ser A. 44, 1089-1098 (2001) (in Chinese)

\section{Submit your manuscript to a SpringerOpen ${ }^{\circ}$ journal and benefit from:}

- Convenient online submission

Rigorous peer review

- Open access: articles freely available online

- High visibility within the field

- Retaining the copyright to your article

Submit your next manuscript at $\gg$ springeropen.com 